\title{
Domestication de nouvelles espèces et développement durable de la pisciculture
}

\author{
Pascal Fontaine ${ }^{1}$ \\ Marc Legendre ${ }^{2}$ \\ Marc Vandeputte ${ }^{3}$ \\ Alexis Fostier ${ }^{4}$ \\ 1 UR AFPA \\ Nancy-Université \\ 2, avenue de la Forêt-de-Haye \\ BP 172 \\ 54505 Vandœuvre-Lès-Nancy \\ France \\ <pascal.fontaine@|sa-man.uhp-nancy.fr> \\ 2 IRD \\ UR 175, Gamet \\ 361, rue J.-F.-Breton \\ BP 5095 \\ 34196 Montpellier cedex 05 \\ France \\ $<$ Marc.Legendre@ird.fr> \\ 3 Ifremer \\ GDR Inra-Ifremer \\ Amélioration génétique des poissons \\ Palavas-les-Flots \\ France \\ $<$ Marc.Vandeputte@jouy.inra.fr> \\ ${ }^{4}$ Inra \\ UR 1037 SCRIBE, IFR 140 \\ 35042 Rennes cedex \\ France \\ <fostier@rennes.inra.fr>
}

\begin{abstract}
Résumé
Il est aujourd'hui acquis que le développement de la pisciculture permet de compenser la stagnation des débarquements de la pêche face à une demande du marché en constante croissance. Ce développement s'accompagne d'une diversification des espèces élevées. Une diversification reposant sur la domestication d'espèces autochtones aux zones d'élevage concernées permettrait de limiter certains risques environnementaux tout en s'adaptant mieux à des marchés locaux. Cela peut aussi favoriser une économie mieux intégrée aux territoires. Dans ce contexte, la mise en place de méthodes génériques de domestication est indispensable à une rationalisation technique et économique de la diversification des espèces d'élevage.
\end{abstract}

Mots clés : développement durable ; diversification ; domestication ; pisciculture.

Thèmes : productions animales ; pêche et aquaculture ; économie et développement rural.

\section{Abstract \\ Domestication of new species and sustainable development in fish culture}

How may fish domestication participate in sustainable development of aquaculture? The development of fish farming currently compensates for the stagnation of fishery catches while the market demand continues to increase. The development of fish farming relies on an active diversification of farmed fish species. A diversification based on the farming of native species could reduce the environmental impact of fish culture and could better fit to needs of local markets. Such development could also favour a more integrated local economy. In this context, the building of a generic approach for multi-species domestication is required to optimise the use of the technical and financial means available for fish culture diversification.

Key words: diversification; domestication; fish culture; sustainable development.

Subjects: animal productions; fishing and aquaculture; economy and rural development. e développement quantitatif de la production piscicole mondiale repose majoritairement sur la généralisation de l'élevage de quelques espèces domestiquées, sans attention particulière sur leur origine géographique, et sur la base de critères essentiellement économiques et techniques. Un quart de la production chinoise, mondialement la plus importante, reposerait sur des espèces introduites (Shelton et Rothbard, 2006). Pour les autres pays d'Asie, la contribution des espèces allochtones dépasserait
$42 \%$ de la production piscicole d'eau douce (De Silva et al., 2006). En Amérique latine, en dépit de la grande richesse spécifique de l'ichtyofaune locale, les espèces de poissons introduites contribuaient encore récemment à plus de $96 \%$ de la production piscicole totale sur ce continent, selon les statistiques de la FAO (Garibaldi, 1996). L'exemple le plus récent de cette démarche est l'introduction du saumon atlantique (Salmo salar) au Chili (Bjørndal, 2001). Cependant, cette introduction massive d'espèces non 
indigènes pour le développement aquacole pose des problèmes environnementaux qui seront précisés plus loin (Buschmann et al., 2006). Constatant la tendance à la diversification dans la production aquacole, une démarche alternative reposant sur le développement d'élevages d'espèces locales est explorée. Celle-ci nécessite cependant de reconsidérer les méthodes de domestication des poissons, aujourd'hui longues et coûteuses.

\section{Pourquoi et quelles nouvelles espèces domestiquer en pisciculture?}

\section{Répondre à la demande et assurer un développement économique mieux intégré aux territoires}

La domestication de nouvelles espèces de poissons, inhérente à la diversification aquacole, est une question récurrente. Cela est un phénomène historiquement récent puisqu'il a été estimé que $97 \%$ de toutes les espèces aquatiques mises en culture ont été domestiquées depuis le début du $\mathrm{xx}^{\mathrm{e}}$ siècle (Duarte et al, 2007). Ainsi, les statistiques de la FAO (2007), couvrant la période 1950-2005, révèlent une croissance constante, de 43 à 219, du nombre d'espèces de poissons utilisées en aquaculture (figure 1), même si la production croît plus vite que le nombre d'espèces élevées, du fait de la large diffusion de quelques espèces dominantes. Parmi les 33 espèces de poissons donnant lieu à une production supérieure à 100000 tonnes en 2005 (c'est-à-dire de l'ordre de la production canadienne ou de deux fois celle de la France), un tiers n'était pas présent dans la production aquacole il y a 60 ans, et près d'un quart il y a 40 ans, ce qui traduit le fait que le développement aquacole des dernières décennies s'est appuyé sur l'élevage de nouvelles espèces. Et encore, ne sont pas prises en compte ici les nombreuses espèces visant le marché de l'aquariophilie en plein développement depuis les années 1990 (Lecchini et al., 2006).
Cette diversification, en termes d'espèces produites, est encouragée par la Commission Européenne (2002) et la FAO (2006). Elle correspond à des situations et à des objectifs variés :

- la nécessité de supprimer la capture de juvéniles sauvages destinés au grossissement en maîtrisant le cycle biologique complet des poissons concernés (cas de l'anguille, Anguilla sp., et du thon, Thunnus sp.) ;

- le passage d'espèces d'un statut " haut de gamme "à un statut de produit de masse (comme le saumon atlantique, Salmo salar) libérant ainsi des segments de marché pour la première catégorie ; - la recherche d'espèces à très fort potentiel de croissance permettant de réduire les coûts de production et les risques associés à la durée des cycles de grossissement (cas du cobia, Rachycentron canadum, et de plusieurs espèces de poissons-chats) ;

- l'attention portée à des espèces à haute valeur symbolique dont les ressources halieutiques s'épuisent dramatiquement (cas de la morue, Gadus morbua, du Pangasius gigas dans le Mékong, de l'Arapaima gigas en Amazonie) ou, plus généralement, à des espèces appréciées mais aux ressources halieutiques limitées (cas du turbot, Psetta maxima) ;

- le besoin de couvrir des marchés traditionnels (marchés de niche) pour lesquels la demande n'est pas satisfaite (cas de la perche commune, Perca fluviatilis) ;
- la diversification de systèmes de production cherchant à intégrer davantage de critères de durabilité comme l'élevage d'espèces locales, d'espèces peu consommatrices de protéines animales ou encore l'optimisation de l'utilisation des intrants par la polyculture ;

- le souhait d'une diversité de choix du consommateur ;

- le besoin de s'adapter aux fluctuations de la demande et du prix des produits;

- la recherche d'espèces pouvant être élevées dans des environnements particuliers (cas des milieux estuariens) ou dans des conditions difficiles (par exemple poissons à respiration aérienne pour des milieux pauvres en oxygène). Par ailleurs, la domestication d'une nouvelle espèce peut être liée à la quête de l'espèce "miracle " par les producteurs et à leur envie d'expérimenter.

Ainsi, outre les avantages économiques tirés de performances zootechniques originales, une diversification aquacole rationnelle peut répondre à des demandes du marché tout en permettant de développer des élevages dont les impacts environnementaux seront limités du fait d'une meilleure intégration aux territoires (espèces indigènes), ou pourront même atténuer les effets négatifs d'autres activités (réduction de la pression de pêche). Cette diversification, lorsqu'elle repose sur l'identification des espèces autochtones présentant des caractéristiques intéressantes pour l'aquaculture, peut aussi

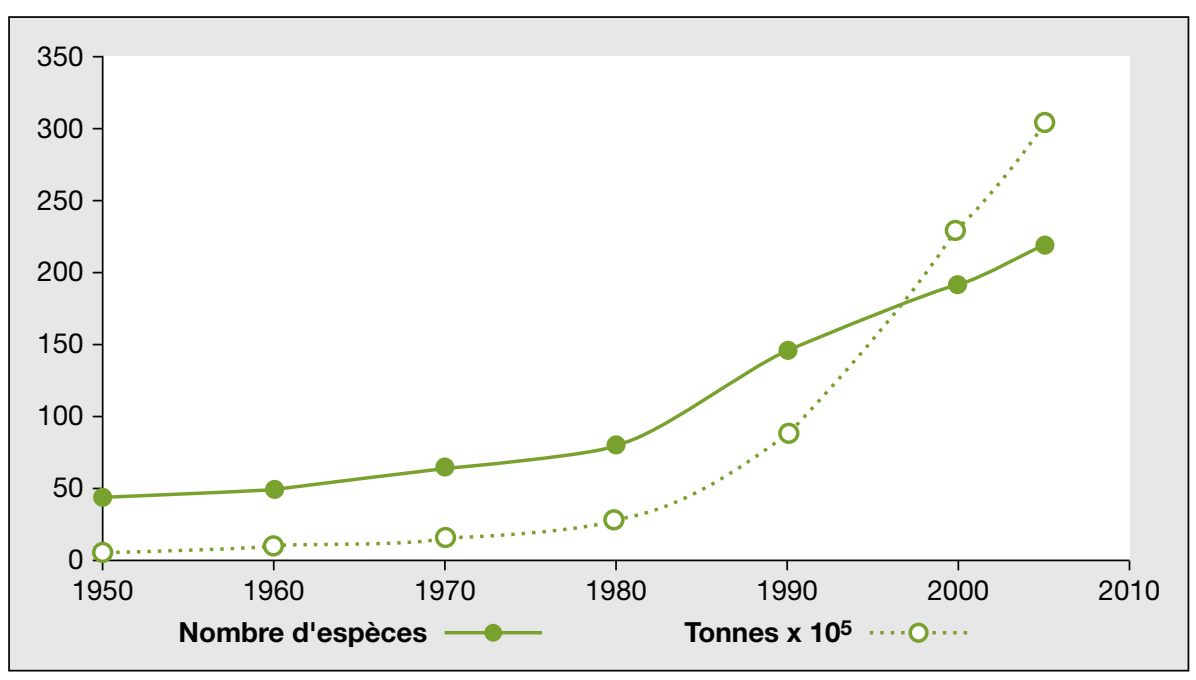

Figure 1. Évolutions, de 1950 à 2005, de la production aquacole (en tonnes) et du nombre d'espèces en élevage.

Figure 1.

Trend in total world finfish aquaculture production (metric tonnes) and number of fish-farmed species between 1950 and 2005. 
conduire à la mise en évidence d'espèces négligées et mal connues révélant un potentiel aquacole supérieur à celui d'une espèce søeur ou d'un genre très voisin utilisé jusqu'alors (Lazard et Legendre, 1994). Tel est le cas par exemple chez les poissons-chats africains, de Chrysichthys nigrodigitatus par rapport à C. maurus ou celui de Heterobranchus longifilis par rapport à Clarias gariepinus (Otémé et al., 1996).

Dans le contexte démographique mondial actuel et par rapport aux potentialités de l'agriculture, le développement de l'aquaculture intégrant la domestication de nouvelles espèces représente un enjeu socioéconomique majeur dont les conséquences potentielles sur l'environnement doivent être attentivement prises en compte (Duarte et al., 2007). Pour le moins, des précautions d'ordre génétique, écologique et sanitaire doivent être prises (Bartley, 1998).

\section{Favoriser un développement durable de la pisciculture par l'utilisation d'espèces autochtones}

L'introduction d'espèces non indigènes peut conduire à des invasions, problème qui demeure la deuxième cause de perte de biodiversité à l'échelle mondiale. Or, le taux d'invasion des écosystèmes aquatiques ne cesse de croitre (Leppäkoski et Olenin, 2000). Les espèces invasives ont la capacité de tolérer une large gamme de conditions, de se reproduire rapidement et efficacement, ce qui induit de nombreux effets sur les écosystèmes envahis : pressions de compétition et de prédation, apports de pathogènes, modifications de l'habitat et du fonctionnement des écosystèmes (Gido et al., 2003).

Cette compétition interspécifique a des conséquences écologiques négatives difficiles à mettre en évidence, en particulier sur la structuration des communautés et la répartition des ressources d'un écosystème (Lodge, 1993). À titre d'exemple, on peut citer les effets de l'introduction du gobie à tache noire (Neogobius melanostomus), originaire de la région de Ponto-Caspian (Caucase) et introduit en mer Baltique, sur l'utilisation de l'habitat et des ressources alimentaires d'une espèce indigène, le flet (Platichtbys flesus) (Karlson et al., 2007). Il s'agit là d'un exemple de compétition entre des espèces de familles différentes, mais de telles compétitions existent aussi entre espèces d'une même famille. Les introductions (1951-1954) de quatre espèces non indigènes de tilapias dans le lac Victoria ont ainsi induit des compétitions avec les espèces indigènes de cichlidés, Oreochromis esculentus et $O$. variabilis (Goudswaard et al., 2002). Ces deux dernières espèces auraient été remplacées par O. niloticus, plus compétitive (opportunisme alimentaire, succès reproducteur supérieur, agressivité, etc.). Des observations similaires ont été faites dans d'autres milieux africains, comme les lacs Ikimba, Ihema et Luhondo, et la rivière Kafue (De Vos et al., 1990 ; Schwank, 1995).

Des exemples de prédation, consécutifs à l'introduction d'espèces non indigènes, ont également été relatés. En milieu dulcicole tempéré (nord de l'Espagne), l'introduction d'un salmonidé, la truite arcen-ciel Oncorbynchus mykiss, a fortement affecté les peuplements d'amphibiens indigènes, notamment les urodèles (Pilliod et Petterson, 2001 ; Denoëel et al., 2005). En milieu tropical (île d'Hawai), on a pu mettre en évidence une prédation due à deux poecilidés, la gambusie (Gambusia affinis) et la molliénésie à voilure (Poecilia latipinna), introduits dans le cadre de programmes de lutte biologique contre les moustiques, sur les populations natives d'odonates (Courtney et Meffe, 1989). Certaines espèces d'odonates ont disparu.

L'introduction d'espèces allochtones s'accompagne souvent du transfert et de la multiplication d'agents pathogènes qui ont un impact négatif et immédiat sur la dynamique des populations hôtes (Barber et al., 2000). On peut citer l'exemple d'Anguillicola crassus, nématode parasite de l'anguille japonaise (Anguilla japonica) et endémique d'Asie du Sud et qui a été introduit accidentellement en Europe à la fin du $\mathrm{xx}^{\mathrm{e}}$ siècle (Koops et Hartmann, 1989). Si l'anguille japonaise semble peu sensible à ce parasite, cela n'a pas été le cas de l'anguille européenne (Anguilla anguilla) (Tesch, 1989). L'introduction de la truite arc-en-ciel (Oncorhynchus mykiss) et du saumon atlantique au sud du Chili a également conduit à la diffusion d'agents pathogènes non indigènes (Carvajal et al., 1998). L'introduction d'espèces peut aussi générer des perturbations des écosystèmes et des habitats comme cela a été démontré pour l'introduction de la carpe commune Cyprinus carpio en Australie (Koehn, 2004) et au Mexique (Hinojosa-Garo et Zambrano, 2004), d'amours blancs (Ctenopharyngodon idella) en Floride (Han- lon et al., 2000), de la truite de mer (Salmo trutta trutta) en Nouvelle-Zélande (Huryn, 1998), du brochet (Esox lucius) au Canada (Elser et al., 2000) ou de la perche du Nil (Lates niloticus) dans les lacs africains Kyoga et Victoria (Seehausen et al., 1997). Les introductions d'espèces modifient les flux d'énergie et de nutriments, et par conséquence le fonctionnement global de l'écosystème.

Enfin, lors de la mise en élevage d'une nouvelle espèce, il faut également considérer les risques de flux géniques intraspécifiques associés au transfert de populations très différenciées génétiquement, car initialement isolées géographiquement. Ce risque existe, par exemple, entre les populations de Pangasius djambal des îles de Sumatra et de Bornéo en Indonésie dont la forte différenciation génétique a été démontrée (Gustiano et al., 2003).

Cette courte synthèse montre clairement qu'il existe de réels risques environnementaux dans l'introduction d'espèces non indigènes. De plus, leurs impacts réels nécessitent un temps assez long pour leur évaluation et, lorsqu'un impact environnemental est observé, il est généralement trop tard pour mettre en ouvre des moyens de lutte, si tant est que ceuxci existent. Cependant, toutes les introductions d'espèces n'ont pas systématiquement été associées à des expériences négatives. L'introduction de la truite arcen-ciel en Europe à des fins d'élevage n'a globalement pas posé de problèmes majeurs, la question se posant davantage pour des déversements volontaires dans un milieu naturel de nombreux individus à des fins de pêche récréative. De même, selon De Silva et al., (2006), l'introduction du tilapia en Asie a donné lieu au développement sur ce continent d'une production très élevée sans occasionner de désordre profond sur les écosystèmes. Les tilapias sont pourtant généralement considérés comme des espèces hautement invasives, susceptibles d'avoir un fort impact sur la biodiversité locale du fait de leur prolificité et de leur adaptabilité à des environnements et ressources trophiques variées. Canonico et al., (2005) présentent plusieurs exemples de tels impacts négatifs pour différentes régions du monde où des tilapias ont été introduits. Malgré ces exceptions apparentes, il semblerait donc en général plus logique de domestiquer des espèces et souches locales.

On ne peut cependant en conclure que la domestication d'espèces locales soit 
toujours sans risques pour les populations naturelles. En effet, mis à part le cas de certains élevages en circuit fermé, les autres sont toujours en contact avec les eaux libres et, à la faveur d'un accident (inondation, tempête, rupture de filets), on peut être quasi-certain que des poissons d'élevage se retrouveront dans le milieu naturel. Or, la domestication s'accompagne de modifications génétiques des populations via la sélection volontaire et involontaire, la relaxation de la sélection naturelle et la dérive génétique (Vandeputte et Launey, 2004). Les animaux domestiqués acquièrent des caractères favorables à l'élevage (forte croissance, maturation tardive, résistance aux maladies, etc.) et peuvent perdre des caractéristiques essentielles à la vie dans le milieu naturel, comme les comportements antiprédateur (Berejikian, 1995) et reproducteur (Fleming et al., 1996), pouvant aboutir à une forte réduction du succès reproducteur dans le milieu naturel (McGinnity et al., 2004; Araki et al., 2007 ; Weir et al., 2004). Cette diminution de caractéristiques essentielles à la survie en milieu naturel, et donc à la valeur adapative des populations, fait que de nombreux auteurs considèrent que le relâcher d'animaux domestiqués, même indigènes, dans le milieu naturel pourrait mettre en danger les populations sauvages (Lynch et O'Hely, 2001; McGinnity et al., 2003 ; Zaporozhets et Zaporozhets, 2004). Cependant, dans certains cas les populations sauvages, bien que soumises au flux de gènes " domestiques " montrent une résilience très forte, ce qui aboutit à recommander en priorité l'élevage d'espèces indigènes plutôt qu'exotiques, avec toutefois la nécessité d'un suivi des effets des échappements sur les populations naturelles (Utter et Epifanio, 2002).

\section{Comment domestiquer de nouvelles espèces en pisciculture?}

Les premiers élevages de poissons remonteraient à plus de 4000 ans, et les premiers témoignages écrits d'une véritable démarche zootechnique en la matière (Traité de Fan Li) datent de 2500 ans (Billard, 2005). Ce sont de fait les carpes, les espèces domestiquées de longue date (Balon, 2004), qui représentent les plus fortes productions à l'heure actuelle (61\% de la production mondiale). Un élevage rationalisé va viser différents objectifs de production conduisant à des choix se traduisant dans des pratiques zootechniques et dans des schémas de sélection plus ou moins élaborés. Ces objectifs évoluent, et avec eux les conditions d'élevage et leurs facteurs environnementaux naturels ou artificiels, mais cela de manière plus ou moins contrôlée en induisant aussi des sélections non intentionnelles (Glover et al., 2004). Il existe, de fait, un continuum de domestication, sachant qu'aucune espèce animale ne peut être considérée comme définitivement domestiquée (Denis, 2004). Une dichotomie trop stricte entre sauvage et domestiqué conduit à négliger une phase de transition entre ces deux états, dont la connaissance est pourtant indispensable à la compréhension du processus de domestication (Dobney et Larson, 2006). Cette phase intermédiaire est d'autant plus intéressante qu'on remarque souvent une différenciation rapide des caractéristiques de populations mises en élevage par rapport à des congénères sauvages (Araki et al., 2007 ; Pearsons et al., 2007), et que justement beaucoup d'espèces de poissons n'en sont qu'aux premières générations de domestication, et donc au cœur de cette phase de transition. Dépasser une approche empirique de cette première phase (essais et erreurs après mise en élevage) pour développer des approches génériques représente un défi majeur pour réussir et optimiser la domestication de nouvelles espèces.

Ces méthodes peuvent s'appuyer, entre autres, sur les apports récents de la génétique moléculaire, de la génomique ainsi que de la bio-informatique dédiée aux études phylogéniques (Fostier et Jalabert, 2004). Ainsi, les données en nombre croissant sur les génomes de poisson permettent de construire, à l'aide des outils de la bio-informatique, de nouvelles classifications phylogénomiques, qu'elles soient basées sur le polymorphisme de marqueurs neutres (microsatellites : répétitions de di-, tri- ou tétranucléotides ; SNP (Single Nucleotide Polymorphism) : polymorphisme mononucléotidique) ou sur celui de gènes (Reed et al., 2002 ; Maes et al., 2006 ; Thai et al., 2007). Ces classifications, associées à des distances génétiques, peuvent aider à prévoir des similarités biologiques entre espèces et donc à extrapoler les connaissances de l'une à l'autre. Parallèlement, l'établissement de classifications reposant sur des traits d'histoire de vie (Teletchea et al., 2007a) permettra d'appréhender les interactions génotype-milieu et les spécialisations écologiques, en fixant les limites d'extrapolations réalisées sur des bases uniquement génétiques. Par ailleurs, les outils de génotypage permettent aussi de mieux appréhender la variabilité génétique de populations fondatrices d'élevages et son évolution avec le processus de domestication (Porta et al., 2007). Enfin, l'analyse de la présence, de la nature et de l'expression de certains gènes, ou familles de gènes, importants dans la mise en œuvre d'une fonction biologique, peut aider à comprendre, pour les orienter, les processus de domestication. Des analyses de gènes candidats mais aussi de transcriptomes peuvent être développés. On peut citer, comme exemple, les ARN messagers maternels de l'œuf impliqués dans les premiers stades de développement embryonnaire et dont la présence va dépendre de l'histoire des génitrices (Bonnet et al., 2007). Des caractéristiques comportementales ou de réaction au stress, particulièrement importantes lors du processus d'adaptation, ont fait aussi l'objet de travaux récents ciblés sur des gènes candidats (Wright et al., 2006) ou des profils de transcrits en grand nombre (Cairns et al., 2008). L'importante composante génétique de la domestication implique des processus de consanguinité, de dérive génétique et de sélection (Mignon-Grasteau et al., 2005). Un mauvais choix de la population fondatrice ou une mauvaise gestion des premières générations (en particulier si les effectifs efficaces de géniteurs sont insuffisants - ce qui est fréquent pour une espèce à l'élevage encore mal maîtrisé) peuvent conduire à une forte réduction de la variabilité génétique d'origine, limitant le potentiel de sélection disponible pour la domestication (Agnèse et al., 1995 ; Vandeputte et Launey, 2004 ; Porta et al., 2007). Le phénotype qui intéresse l'aquaculteur dépendra aussi des interactions génotype-environnement et de la plasticité phénotypique influencée par l'histoire de vie des populations (Duponchelle et Legendre, 2001 ; Huntingford, 2004). Quand les interactions génotypemilieu existent, on peut par ailleurs douter de la possibilité de conduire une domestication universelle, pour tout système d'élevage (Vandeputte et Launey, 2004). Toutefois, la définition de groupes fonctionnels (Teletchea et al., 2007a), 
basés sur la convergence des traits de vie des espèces pour une fonction biologique donnée ou le cycle de vie, pourrait permettre la conception d'une approche générique de la domestication. Une fois les groupes fonctionnels constitués, cette dernière pourrait suivre les étapes suivantes: identification d'espèces modèles pour chaque groupe, modélisation du déterminisme multifactoriel des performances des espèces modèles (approches multidisciplinaires et multidimensionnelles) et validation du sens des groupes fonctionnels (extrapolation des connaissances). Certaines caractéristiques biologiques sont par ailleurs de bons indicateurs des performances zootechniques susceptibles de s'exprimer en milieu d'élevage. La taille maximale d'un poisson constitue ainsi un indicateur de sa vitesse de croissance; les plus grandes espèces sont très généralement celles qui possèdent le meilleur potentiel de croissance (Legendre et Albaret, 1991). Le maintien d'une forte variabilité génétique, par des plans de croisement adaptés, éventuellement mieux contrôlés par l'utilisation de marqueurs génétiques, aide à garder un potentiel d'adaptation à différents milieux. Il devient aussi envisageable de s'intéresser, de manière ciblée, au polymorphisme de gènes impliqués dans des caractères utiles à la domestication (Vandeputte et Prunet, 2002). D'un point de vue expérimental, on peut utiliser des poissons modèles, comme le poisson zèbre Danio rerio, pour étudier des processus génétiques de domestication (Robison et Rowland, 2005). On peut également rationaliser l'étude des effets des facteurs du milieu sur les phénotypes en développant des plans d'expérimentation multifactoriels (Wang et al., 2006). Enfin, la construction d'arbres phylogéniques basés sur des caractéristiques écobiologiques peut aider à faire le choix de conditions d'élevage et de biotechnologies adaptées pour une espèce donnée, cela par extrapolation des connaissances acquises au sein de l'ensemble de son groupe (Teletchea et al., 2007b). Ces arbres donnent également une image objective et synthétique de la diversité des phénotypes disponibles.

Ces réflexions portent essentiellement sur les aspects biologiques et zootechniques de la domestication. Il reste que le choix de l'espèce visant à optimiser la diversification devra aussi prendre en compte les contraintes environnementales et socioéconomiques de la zone aquacole considérée (Hem et al., 1994 ; Tisdell, 2007).

\section{Conclusion}

Les choix et les méthodes pour la domestication de nouvelles espèces sont un réel enjeu pour un développement durable de la pisciculture. Il doit exister des alternatives au seul transfert d'espèces non indigènes choisies sur des critères de rentabilité à court terme (Shelton et Rothbard, 2006). En s'appuyant sur les demandes ciblées des populations locales, où les espèces consommées sont souvent associées à une valeur du territoire concerné (exemple de la consommation de perche en Suisse associée aux lacs alpins) et peuvent avoir une valeur patrimoniale mais aussi diététique dans des zones de sousnutrition (Roos et al., 2007), la domestication d'espèces indigènes limiterait les risques environnementaux liés à l'introduction d'espèces ou de populations exotiques (principe de précaution). On peut aussi noter que des aquasystèmes, comme la rizipisciculture, relevant de "Systèmes ingénieux du patrimoine agricole d'importance mondiale" (concept FAO du Système ingénieux du patrimoine agricole d'importance mondiale, le GIAHS), intègrent l'utilisation d'espèces indigènes (Lu et Li, 2006).

Toutefois, la domestication d'une espèce nouvelle, qui plus est, choisie sur d'autres critères que sa facilité d'élevage, est une opération très coûteuse. Le nombre d'espèces domesticables étant élevé, il est nécessaire de développer une approche générique en rationalisant les connaissances actuelles et en identifiant les convergences (regroupements fonctionnels). A terme, une telle démarche pourrait permettre un accès plus rapide à des espèces d'intérêt aquacole susceptibles de mieux s'intégrer dans un contexte socioéconomique et environnemental donné.

\section{Références}

Agnèse JF, Otémé ZJ, Gilles S. Effects of domestication on genetic variability, fertility, survival and growth rate in a tropical siluriform: Heterobranchus longifilis Valenciennes 1840. Aquaculture 1995 ; 131 : 197-204.

Araki H, Cooper B, Blouin MS. Genetic effects of captive breeding cause a rapid, cumulative fitness decline in the wild. Science $2007 ; 318$ : 100-3.

Balon EK. About the oldest domesticates among fishes. J Fish Biol 2004 ; 65 : 1-27.

Barber I, Hoare D, Krause J. Effects of parasites on fish behaviour: a review and evolutionary perspective. Rev Fish Biol Fish 2000; 10: 131-65.
Bartley DM. A precautionary approach for the introduction of new species in aquaculture. In : Enne G, Greppi GF, eds. New species for Mediterranean aquaculture. Amsterdam: Elsevier, 1998.

Berejikian BA. The effects of hatchery and wild ancestry and experience on the relative ability of steelhead trout fry (Oncorhynchus mykiss) to avoid a benthic predator. Can J Fish Aquat Sci $1995 ; 52: 2476-82$.

Billard R. Introduction à l'aquaculture. Paris : Lavoisier, 2005.

Bjørndal T. The competitiveness of the Chilean salmon aquaculture industry. Working Paper 2001-37. Bergen (Norvège): SNF/Centre for Fisheries Economics Series, 2001. http://hdl. handle.net/2330/930.

Bonnet E, Bobe J, Fostier A. Microarray-based analysis of fish egg quality after natural or controlled ovulation. BMC Genomics 2007 ; 8 : 55.

Buschmann $\mathrm{AH}$, Riquelme VA, HernandezGonzalez MC, et al. A review of the impacts of salmonid farming on marine coastal ecosystems in the southeast Pacific. ICES J Mar Sci $2006 ; 63: 1338-45$.

Cairns MT, Johnson MC, Talbot AT, et al. A cDNA microarray assessment of gene expression in the liver of rainbow trout (Oncorhynchus mykiss) in response to a handling and confinement stressor. Comp Biochem Physiol D- Genomics Proteomics 2008 ; 3 : 51-66.

Canonico GC, Arthington A, McCrary JK, et al. The effects of introduced tilapias on native biodiversity. Aquatic. Conserv Mar Freshw Ecosyst $2005 ; 15$ : 463-83.

Carvajal J, González L, George-Nascimento M. Native sea lice (Copepoda: Caligidae) infestation of salmonids reared in netpen systems in southern Chile. Aquaculture 1998 ; 166 : 241-6.

Commission Européenne. Communication from the Commission to the Council and the European Parliament: a strategy for the sustainable development of European aquaculture. COM 511 final. SI : Commission européenne, 2002.

Courtenay WR, Meffe GK. Small fishes in strange places: a review of introduced poeciliids In: Meffe GK Snelson Jr. FF eds. Ecology and evolution of live-bearing fishes (Poecillidae). New Jersey: Englewood Cliffs, 1989.

Denis. La domestication: un concept devenu pluriel. Inra Prod Anim 2004 ; 17 : 161-6.

Denoël M, Dzukic G, Kalezic ML. Effect of widespread fish introductions on paedomorphic newts in Europe. Conserv Biol 2005; 19 : 162-70.

De Silva S, Nguyen T, Abery NW, et al. An evaluation of the role and impacts of alien finfish in Asian inland aquaculture. Aquat research 2006 ; $37: 1-17$.

De Vos L, Snoeks J, Van den Audenaerde D. The effects of tilapia introductions in lake Luhondo, Rwanda. Environ. Biol Fish 1990; 27 : 303-8.

Dobney K, Larson G. Genetics and animal domestication: new windows on an elusive process. J Zool 2006 ; 269 : 261-71.

Duarte M, Marbá N, Holmer M. Rapid domestication of marine species. Science $2007 ; 316$ : 382-3. 
Duponchelle F, Legendre M. Rapid phenotypic changes of reproductive traits in response to experimental modifications of spatial structure in Nile tilapia, Oreochromis niloticus. Aquat Liv Res 2001 ; 14 : 145-52.

Elser JJ, Sterner RW, Galford AE, et al. Pelagic CNP stochiometry in an eutrophied lake: res ponses to a whole-lake food-web manipulation. Ecosystems (NY Print) 2000 ; 3 : 293-307.

FAO. Fishstat Plus - Universal software for fisheries statistical time series - Aquaculture production: quantities. www.fao.org, 2007.

FAO. Prospective analysis of future aquaculture development. Rome: FAO, 2006.

Fleming IA, Jonsson B, Gross MR, et al. An experimental study of the reproductive behaviour and success of farmed and wild Atlantic salmon (Salmo salar). J Appl Ecol 1996; 33 : 893-905.

Fostier A, Jalabert B. Domestication et reproduction chez les poissons. Prod Anim 2004 $17: 199-204$.

Garibaldi L. List of animal species used in aquaculture. FAO Fisheries Circular, $\mathrm{n}^{\circ} 914$ Rome: FAO, 1996.

Gido KB, Schaefer JF, Pigg J. Patterns of fish invasions in the great plains of North America. Biol Conserv 2003; 118 : 121-31.

Glover KA, Taggart JB, Skaala O, et al. A study of inadvertent domestication selection during start feeding of brown trout families. J Fish Biol $2004 ; 64$ : 1168-78.

Goudswaard PC, Witte F, Katunzi EFB. The tilapiine fish stock of lake Victoria before and after the Nile perch upsurge. J Fish Biol 2002; 60 : 838-56.

Gustiano R, Sudarto, Pouyaud L. How to recognise Pangasius djambal? p. 3-14. In: Slembrouck J., Komarudin O, Maskur, Legendre M, eds. Technical manual for artificial propagation of the Indonesian catfish, Pangasius djambal. Paris: Éditions IRD, 2003.

Hanlon SG, Hoyer MV, Cichra CE, et al. Evaluation of macrophyte control in 38 Florida lakes using triploid grass carp. J Aquat Plant Manage 2000 ; 38: 48-54.

Hem S, Legendre M, Trébaol L, et al. L'aquaculture lagunaire. In : Durand JR, Dufour $P$ Guiral D, Zabi SG, eds. Environnement et ressources aquatiques de Côte-d'lvoire. 2 -les milieux lagunaires. Paris : Éditions IRD, 1994.

Hinojosa-Garro D, Zambrano L. Interactions of common carp (Cyprinus Carpio) with benthic crayfish decapods in shallow ponds. Hydrobio logia $2004 ; 515: 115-22$.

Huntingford FA. Implications of domestication and rearing conditions for the behaviour of cultivated fishes. J Fish Biol 2004; 65 (Suppl. A) : 122-42

Huryn AD. Ecosystem level evidence for topdown and bottom-up control of production in a grassland stream system. Oecologia 1998 $115: 173-83$

Karlson AML, Almqvist G, Skora KE, et al. Indications of competition between nonindigenous round goby and native flounder in the Baltic sea. ICES J Mar Sci 2007 ; 64 : 479-86

Koehn JD. Carp (Cyprinus carpio) as a powerful invader in Australian waterways. Fresh Bio $2004 ; 49$ : 882-94.

Koops H, Hartmann F. Anguillicola-infestations in Germany and German eel imports. J Appl Ichthyol 1989: $5: 41-5$.
Lecchini D, Polti S, Nakamura $Y$, et al. New perspectives on aquarium fish trade. Fish Sci $2006 ; 72: 40-7$.

Lazard J, Legendre M. La pisciculture africaine : enjeux et problèmes de recherche. Cah Agric 1994 ; 3 : 83-92.

Legendre M, Albaret JJ. Maximum observed length (MOL) as an indicator of growth rate in tropical fishes. Aquaculture 1991; 94 : 327-41.

Leppäkoski E, Olenin S. Non-native species and rates of spread: lessons from the brackish Baltic Sea. Biol Invasions $2000 ; 2$ : 151-63.

Lodge DM. Biological invasions - lessons for ecology. Tends Ecol Evol 1993; 8 : 133-7.

Lu JB, Li X. Review of rice-fish-farming systems in China: one of the Globally Important Ingenious Agricultural Heritage Systems (GIAHS). Aquaculture 2006 ; 260 : 106-13.

Lynch M, O'Hely M. Captive breeding and the genetic fitness of natural populations. Conserv Genet $2001 ; 2$ : 363-78.

Maes GE, Pujolar JM, Raeymaekers JAM, et al. Microsatellite conservation and Bayesian individual assignment in four Anguilla species. Mar Ecol Prog Ser 2006 ; 319 : 251-61.

McGinnity $P$, Prodhöl P, Ferguson A, et al. Fitness reduction and potential extinction of wild populations of Atlantic salmon, Salmo salar, as a result of interactions with escaped farm salmon. Proc R Soc Lond B Biol Sci $2003 ; 270$ : 2443-50.

McGinnity $\mathrm{P}$, Prodohl $\mathrm{P}$, Maoileidigh $\mathrm{O}$, et al. Differential lifetime success and performance of native and non-native Atlantic salmon examined under communal natural conditions. J Fish Biol 2004 ; 65 : 173-87.

Mignon-Grasteau S, Boissy A, Bouix J, et al. Genetics of adaptation and domestication in livestock. Livest Prod Sci 2005 ; 93 : 3-14.

Otémé Z, Hem S, Legendre M. Nouvelles espèces de poissons-chats pour le développement de la pisciculture africaine. In: Legendre M, Proteau J-P, eds. The biology and culture of catfishes. Aquat Living Resour 1996; HS (9): 207-17.

Pearsons TN, Fritts AL, Scott JL. The effects of hatchery domestication on competitive dominance of juvenile spring Chinook salmon (Oncorhynchus tshawytscha). Can J Fish Aquat Sci 2007 ; 64 : 803-12.

Pilliod DS, Peterson CR. Local and landscape effects of introduced trout on amphibians in historically fishless watersheds. Ecosystems (NY Print) $2001 ; 4: 322-33$.

Porta J, Porta JM, Cañavate P, et al. Substantial loss of genetic variation in a single generation of Senegalese sole (Solea senegalensis) culture: implications in the domestication process. J Fish Biol 2007 ; 71 : 223-34.

Reed DL, Kent E, Carpenter KE, deGravelle MJ. Molecular systematics of the Jacks (Perciforms: Carangidae) based on mitochondrial cytochrome $B$ sequences using parsimony, likelihood, and Bayesian approaches. Mol Phylogen Evol $2002 ; 23$ : 513-24.

Robison BD, Rowland W. A potential model system for studying the genetics of domestication: behavioural variation among wild and domesticated strains of zebra Danio (Danio rerio). Can J Fish Aquat Sci 2005 ; 62 : 2046-54.
Roos N, Wahab MA, Hossain MAR, et al. Linking human nutrition and fisheries: incorporating micronutrient-dense, small indigenous fish species in carp polyculture production in Bangladesh. Food Nut Bull 2007; 28: S 280-93.

Schwank EJ. The introduced Oreochromis niloticus is spreading on the Kafue floodplain Zambia. Hydrobiologia 1995 ; 315 : 143-7.

Seehausen O, Witte F, Katunzi EF, et al. Patterns of the remnant cichlid fauna in Southern lake Victoria. Conserv Biol 1997; 11 : 890-904.

Shelton WL, Rothbard S. Exotic species in global aquaculture: a review. Isr J Aqua Bamidgeh $2006 ; 58: 3-28$

Teletchea F, Fostier A, Kamler E, et al. Comparative analysis of reproductive strategies of European freshwater fishes: applications to the domestication of new species in aquaculture. 8th International Symposium on Reproductive Physiology of Fish, June 3-8, SaintMalo, France. 2007a

Teletchea F, Fostier A, Le Bail PY, et al. STORE FISH: a new database dedicated to the reproduction of temperate freshwater teleost fishes. Cybium 2007 ; $31: 227-35$

Tesch FW. Changes in swimming depth and direction of silver eels (Anguilla anguilla L.) from continental shelf to deep sea. Aquat Living Resour 1989; 2 : 9-20.

Thai BT, Si VN, Phan PD, Austin CM. Phylogenetic evaluation of subfamily classification of the Cyprinidae focusing on Vietnamese species. Aquat Living Resour 2007 ; 20 : 143-53.

Tisdell C. The environment and the selection of aquaculture species and systems: an economic analysis. In: Leung PS, Lee CS, O'Bryen PJ, eds. Species and system selection for sustainable aquaculture. Ames (lowa); Oxford: Blackwell Publishing, 2007.

Utter F, Epifanio J. Marine aquaculture: genetic potentialities and pitfalls. Rev Fish Biol Fish $2002 ; 12: 59-77$.

Vandeputte $M$, Prunet $P$. Génétique et adaptation chez les poissons: domestication, résistance au stress et adaptation aux conditions de milieu. Inra Prod Anim 2002 ; 15 : 365-71.

Vandeputte M, Launey S. Quelle gestion génétique de la domestication chez les poissons? Inra Prod Anim 2004; 17 : 237-42.

Wang N, Gardeur JN, Henrotte E, et al. Determinism of the induction of the reproductive cycle in female Eurasian perch, Perca fluviatilis: identification of environmental cues and permissive factors. Aquaculture 2006; 261 : 706-14.

Weir LK, Hutchings JA, Fleming IA, et al. Dominance relationships and behavioural correlates of individual spawning success in farmed and wild male Atlantic salmon, Salmo salar. J Anim Ecol 2004; 73 : 1069-79.

Wright D, Nakamichi R, Krause J, et al. QTL analysis of behavioral and morphological differentiation between wild and laboratory zebrafish (Danio rerio). Behav Genet 2006; 36 : 271-84.

Zaporozhets OM, Zaporozhets GV. Interaction between hatchery and wild Pacific salmon in the Far East of Russia: a review. Rev Fish Biol Fish 2004 ; 14 : 305-19. 